\title{
PROGRAMA PARA VISUALIZAÇÃO DE IMAGENS E ESPECTROS ASTRONÔMICOS DO TIPO FITS
}

\section{SOFTWARE FOR VIEWING IMAGES AND ASTRONOMICAL SPECTRA OF TYPE FITS}

Felipe Gonçalves de Toledo; Francisco Assis da Silva; Mário Augusto Pazoti; Leandro Luiz de Almeida; Robson Augusto Siscoutto; Danillo Roberto Pereira

Universidade do Oeste Paulista - UNOESTE, Faculdade de Informática - FIPP, Presidente Prudente - SP. e-mail: danilopereira@unoeste.br

RESUMO - Este trabalho apresenta uma proposta para manipular imagens e espectros astronômicos do tipo FITS, formato comumente usado na astronomia. É gerada uma imagem colorida dos objetos no espaço (estrelas, galáxias, nebulosas, etc.) com o conceito de falsa cor, a partir de três imagens em tons de cinza capturadas com diferentes filtros de comprimento de onda, e realiza breves análises do espectro eletromagnético para se obter algumas informações, como a composição e o movimento do objeto observado. O software foi desenvolvido na linguagem Java com o auxílio de bibliotecas para a manipulação das imagens, gráficos e do arquivo FITS.

Palavras-chave: Imagens astronômicas, FITS, espectros.

ABSTRACT - This work presents a proposal to manipulate astronomical images and spectra of type FITS format commonly used in astronomy. It generates a colored image of objects in space (stars, galaxies, nebulae, etc.) with the concept of false color, from three gray scale images captured with different wavelength filters, and makes brief analysis of the electromagnetic spectrum to obtain some information, such as the composition and movement of the observed object. The software was developed in Java including some libraries to manipulate images, graphics and FITS file.

Keywords: Astronomical images, spectra, FITS.

Recebido em: 09/10/2014

Revisado em: 07/11/2014

Aprovado em: 15/12/2014 


\section{INTRODUÇÃO}

A astronomia é datada bem antes da história de Cristo, aproximadamente 3000 A.C. Ela aparece entre vários povos, como por exemplo, os babilônios, os chineses e egípcios, e naquela época, os astros eram estudados com objetivo práticos, como medir a passagem do tempo (fazer calendários) para prever a melhor época para o plantio e a colheita. Os babilônios, assírios e egípcios também sabiam a duração do ano desde épocas pré-cristãs. Em outras partes do mundo, evidências de conhecimentos astronômicos muito antigos foram deixadas na forma de monumentos, como o de Stonehenge, na Inglaterra, que data de 3.000 a 1.500 A.C. Nessa estrutura, algumas pedras estão alinhadas com o nascer e o pôr do Sol no início do verão e do inverno (OLIVEIRA FILHO, 2004). Sem possuir tecnologia, as deduções eram baseadas apenas nos dados observados do cotidiano, chegando a várias conclusões errôneas sobre como era a Terra (STEINER, 2006).

Por muito tempo acreditou-se que a Terra era o centro do universo, e para que a Terra perdesse o seu posto no centro do universo seria necessária a criação de uma nova física, compatível com o sistema heliocêntrico (Terra orbitando o sol), e, para isso, contribuíram enormemente dois eventos ocorridos no ano de 1609. Na Itália,
Galileu Galilei teve a ideia de apontar uma luneta náutica em direção ao céu, fazendo uma série de observações absolutamente inéditas que trariam grandes dificuldades para os defensores da visão tradicional do cosmos (ITOKAZU, 2009).

Com o passar do tempo, surgiram telescópios mais avançados. Hoje não há mais necessidade de se observar o universo a olho nu. As observações são feitas a partir de imagens e espectros capturados por imensos telescópios e espectrógrafos. A maior fonte de todos esses dados é o Telescópio Espacial Hubble, localizado na órbita da Terra para obter melhores informações e sem interferência da atmosfera terrestre. Todos esses dados capturados são armazenados em um formato padrão, o arquivo FITS (Flexible Image Transport System), que significa sistema de transporte de imagem flexível. Analisando as imagens e espectro é possível descobrir informações como o movimento, temperatura e composição dos objetos observados (TENNYSON, 2005).

São necessárias ferramentas para a leitura do arquivo FITS, como o "SAOImage DS9" ou o "FITS Liberator", sendo que ambos possibilitam a visualização das imagens contidas nesse arquivo. Para a manipulação do espectro usa-se a ferramenta "SpecView".

Tendo em vista esse cenário, buscouse desenvolver uma ferramenta que possibilita trabalhar com imagens e espectro 
em um mesmo ambiente, sem necessidade de duas ferramentas. É possível gerar uma imagem colorida facilmente, e também realiza algumas análises básicas utilizando o gráfico do espectro.

Este trabalho está organizado da seguinte forma: na Seção 2 são apresentados os Conceitos Fundamentais; na Seção 3 é apresentada a Metodologia; na Seção 4 são mostrados os Experimentos e Resultados; na Seção 5 são apresentadas as Conclusões;

\section{CONCEITOS FUNDAMENTAIS}

Nesta seção estão descritas as teorias envolvidas a respeito de imagens astronômicas, de espectro astronômico e do tipo de arquivo FITS, utilizado para armazenar dados astronômicos.

\subsection{Imagem astronômica}

As cores vistas são resultados de como o olho e o cérebro humano percebem diferentes comprimentos de onda de luz na parte visível do espectro eletromagnético. A luz é reduzida para três componentes de cor pelo olho (vermelho, verde e azul). As imagens em cor verdadeira de um objeto são imagens que aparecem para o olho humano exatamente como o objeto é; o céu é azul, a maça é vermelha. A imagem em cor falsa é uma imagem que representa um objeto em cores diferentes das que uma fotografia colorida com cor verdadeira mostraria. O termo falsa cor é usado, na maioria das vezes, para descrever imagens nas quais as cores representam intensidades fora da parte visível do espectro eletromagnético (invisível ao olho humano). Imagens astronômicas são, geralmente, coloridas com falsa cor (CHANDRA, 2013).

Capturar imagens coloridas com o telescópio Hubble é muito mais complexo do que tirar fotos coloridas com uma câmera tradicional. Por um motivo, o Hubble não usa filme colorido, na verdade não usa nenhum filme. Suas câmeras gravam a luz do universo com detectores eletrônicos especiais. Esses detectores não produzem imagens coloridas, produzem imagens em preto e branco (HUBBLE, 2013). Essas imagens sem cor devem ser ajustadas para enfatizar as características individuais ou processos que os cientistas estão interessados, ou apenas melhoradas para fins estéticos. Em momento algum é criado ou modificado algo que já não estava presente na imagem. Colorir uma imagem astronômica envolve uma cuidadosa seleção de cores escolhidas para representar algumas características na imagem, como uma intensidade, energia ou composição química. Com a cor falsa é possível obter informações de intensidade do invisível para o visível por meio da atribuição de cor para imagens individuais. As cores escolhidas, usualmente vermelho, verde e azul, são geralmente mapeadas de acordo com o 
comprimento de onda, de modo que os comprimentos mais longos de luz presente na imagem são mapeados para o vermelho, enquanto que os de comprimento de onda mais curtos se movem por meio do verde e do azul (CHANDRA, 2013).

Os objetos aparecem diferentes em cada comprimento de onda da luz, como mostrado na Figura 1. Para capturar como o objeto aparece em certo comprimento de onda, é feito o uso de filtros que permitem a passagem apenas de uma faixa de comprimento.

As imagens não precisam ser necessariamente obtidas apenas da parte visível, podem ser obtidas a partir de qualquer comprimento de onda de luz, como visto na Figura 1: (a) As duas primeiras imagens representam a emissão de ultravioleta (comprimentos de onda de aproximadamente $300 \mathrm{~nm}$ ), (b) as duas intermediárias são da parte visível do espectro eletromagnético (entre $400 \mathrm{~nm}$ e $700 \mathrm{~nm}$ ) e (c) as três últimas representam a emissão de infravermelho (aproximadamente $1000 \mathrm{~nm})$.

As imagens astronômicas coloridas são geradas pela combinação de imagens em tons de cinza representando diferentes cores de luz, como mostra a Figura 2.

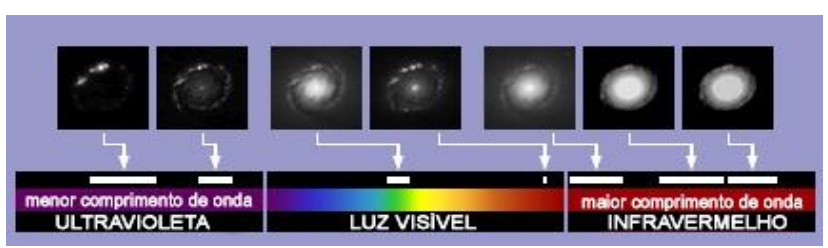

Figura 1. Como a imagem é representada em diferentes comprimentos de onda. Fonte: (HUBBLE, 2013).

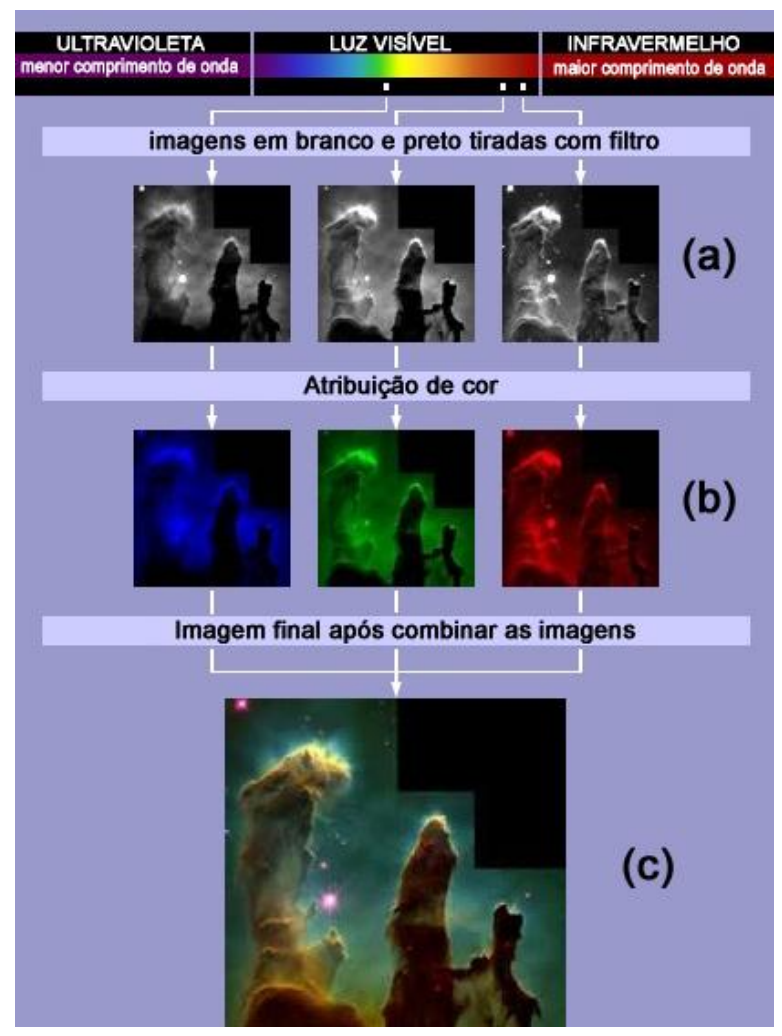

Figura 2. Processo de coloração da imagem. Fonte: (HUBBLE, 2013).

\subsection{Espectro astronômico}

A luz pode ser descrita como ondas que viajam pelo espaço. O comprimento de onda é a distância entre dois picos, como destacados na Figura 3. Diferentes comprimentos de onda de luz visível são vistos como diferentes cores pelos olhos, do comprimento de onda mais comprido para o mais curto, as cores que os olhos vêem são: vermelho, laranja, amarelo, verde, azul e violeta. A luz branca é uma mistura de todas 
as cores, ou em outras palavras, uma mistura de luz com todos os comprimentos de onda (BROSIUS, 1997).

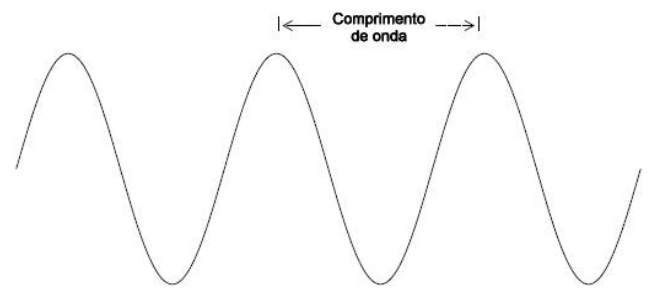

Figura 3. Representação de um comprimento de onda.

Fonte: (BROSIUS, 1997).

Os objetos (estrelas, galáxias, nebulosas, etc.) no espaço emitem mais do que apenas luz visível, eles emitem luz invisível também, que pode ter comprimentos de onda maiores (como o infravermelho, micro-ondas e rádio) ou comprimentos de onda menores (como o ultravioleta, raios-X e raio gama) do que a luz visível. O termo técnico para todas essas formas de luz é radiação eletromagnética. Quando colocadas lado a lado, em ordem crescente ou decrescente de comprimento de onda, as diferentes formas de luz formam o espectro eletromagnético. Um arco íris, que é um espectro de luz visível, é apenas uma pequena parte do espectro eletromagnético (BROSIUS, 1997), como mostrado na Figura 4.

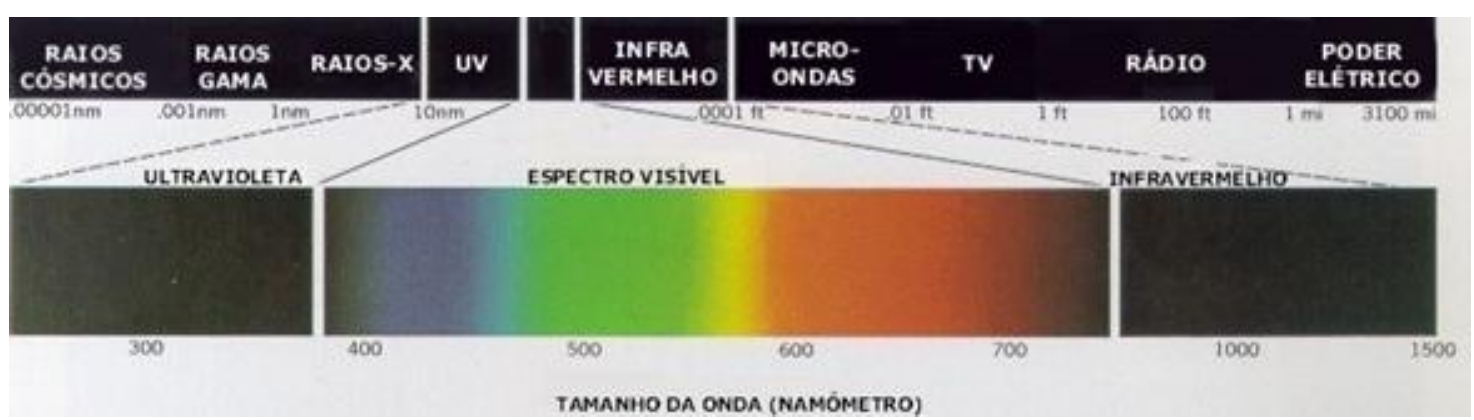

Figura 4. Espectro Eletromagnético.

Fonte: (GONZALEZ, 2009).

Astrônomos medem o brilho da luz através das cores (a luz é separada em diferentes comprimentos de onda, visíveis e não visíveis, com o uso de um prisma) e criam um gráfico com a intensidade de cada comprimento de onda obtido (Figura 5). O gráfico contém muito mais informação do que as imagens, e por meio dele os astrônomos conseguem aprender muito sobre o objeto observado, tal como movimento, composição, e temperatura. 

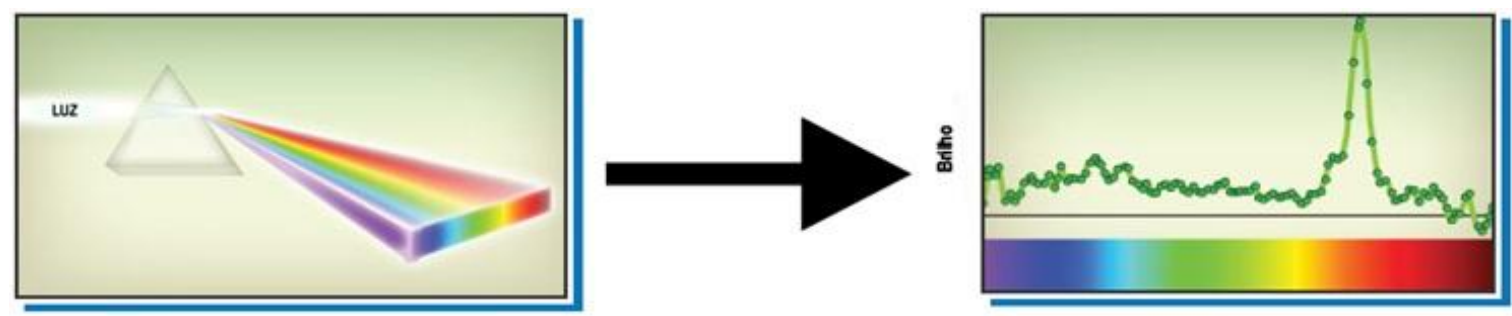

Comprimento de onda

Figura 5. Faixa de luz visível $(400 \mathrm{~nm}-700 \mathrm{~nm})$ sendo transformada em espectro.

Fonte: (HUBBLE, 2013).

Analisando o gráfico do espectro é possível descobrir a composição do objeto a partir da radiação emitida de cada átomo ou íon (Figura 6 (b)). Quanto maior intensidade de emissão em comprimentos de onda menores, mais alta é a temperatura (Figura 6 (a)) e o movimento é analisado pelo efeito Doppler (comprimento de onda deslocado devido ao movimento em relação ao observador, mostrado na Figura 6 (c)) provocado no espectro (TENNYSON, 2005).

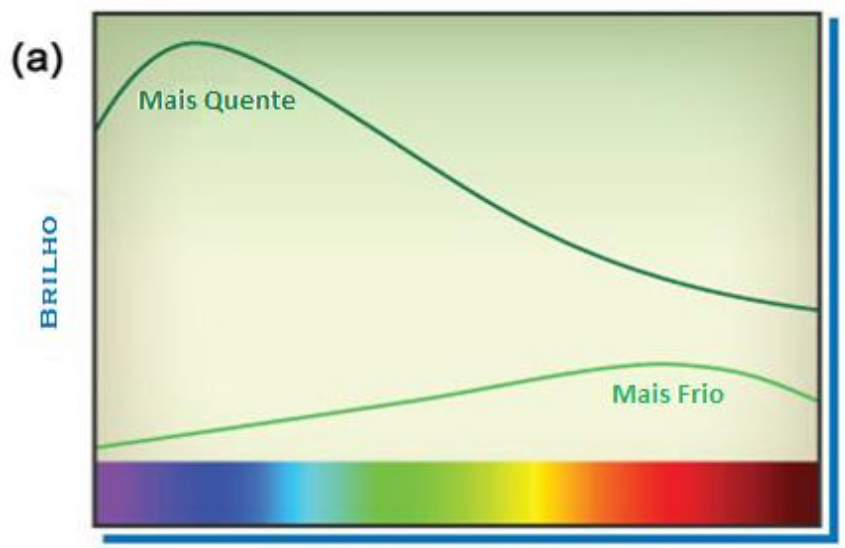

COMPRIMENTO DE ONDA

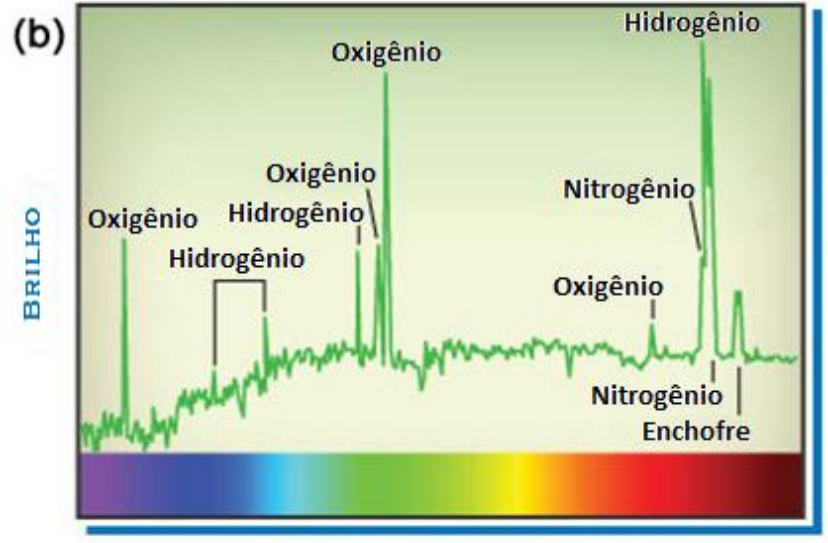

COMPRIMENTO DE ONDA

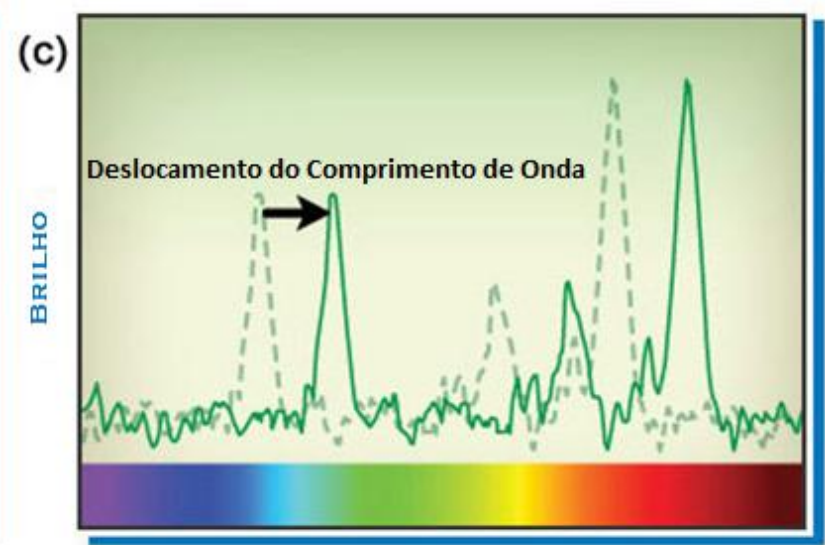

COMPRIMENTO DE ONDA

Figura 6. (a) Temperatura (b) Composição (c) Movimento.

Fonte: (HUBBLE, 2013). 


\subsection{Formato FITS}

Esse formato de arquivo foi criado para transportar e armazenar dados astronômicos de $n$-dimensões e contém um cabeçalho dinâmico que pode ser alterado de acordo com a necessidade de adicionar ou remover parâmetros referentes ao conteúdo do arquivo (WELLS; GREISEN; HAETEM, 1981)

Um arquivo FITS é composto pela imagem ou espectro e por um cabeçalho associado ao mesmo. $\mathrm{O}$ arquivo utiliza $\mathrm{o}$ formato binário para o armazenamento das imagens e $\mathrm{o}$ formato ASCll para 0 armazenamento do cabeçalho, ilustrado pela Figura 7, permitindo aos pesquisadores e máquinas o reconhecimento das informações contidas nesse cabeçalho (NOGUEIRA; VAZ; SOUZA, 2010).

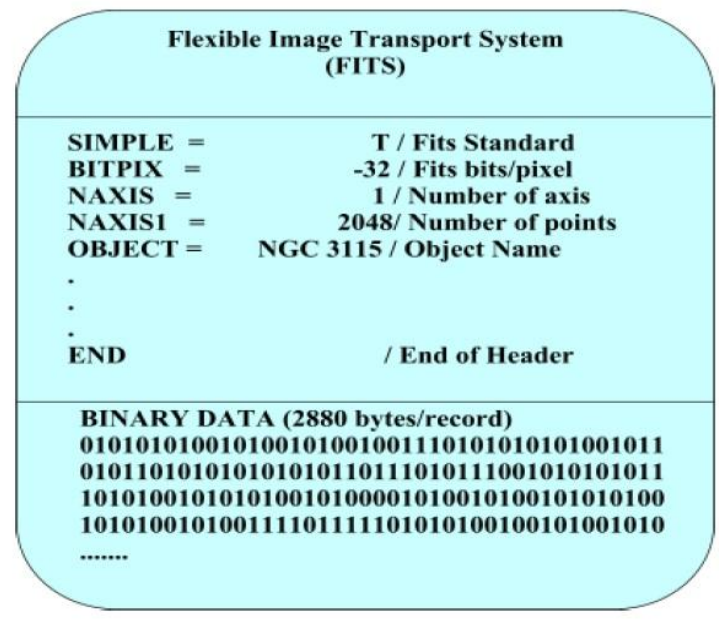

Figura 7. Estrutura de um arquivo FITS. Fonte: (NOGUEIRA; VAZ; SOUZA, 2010).

\section{METODOLOGIA}

Nesta seção são descritas as etapas realizadas para obter os dados da imagem a partir do arquivo FITS, normalização e transformação dos dados para o padrão de cores RGB, equalização para enfatizar detalhes presentes na imagem e a geração da imagem colorida a partir da fusão de três imagens em níveis de cinza capturadas com três diferentes filtros (CHANDRA, 2013). Também são mostrados os passos para obter o gráfico do espectro e como foi feito para realizar algumas análises.

\subsection{Tratamento de imagem astronômica}

Para realizar a leitura do cabeçalho e dos dados referentes à imagem contida no arquivo FITS, foi utilizada a biblioteca Java "nom.tam.fits", disponibilizada pela NASA. Os dados da imagem obtidos estão contidos em uma estrutura de números reais.

É necessário realizar uma normalização dos dados entre 0 e 1 para convertê-los para o modelo de cor RGB. Neste ponto a imagem já pode ser visualizada, como mostrado na Figura 8 (a). Para realçar as características presentes na imagem foi aplicada a equalização de histograma (GONZALEZ, 2009), que realiza uma distribuição no histograma , por meio da biblioteca "ImageJ". Na Figura 8 (b) é mostrado o resultado da equalização. 
(a)
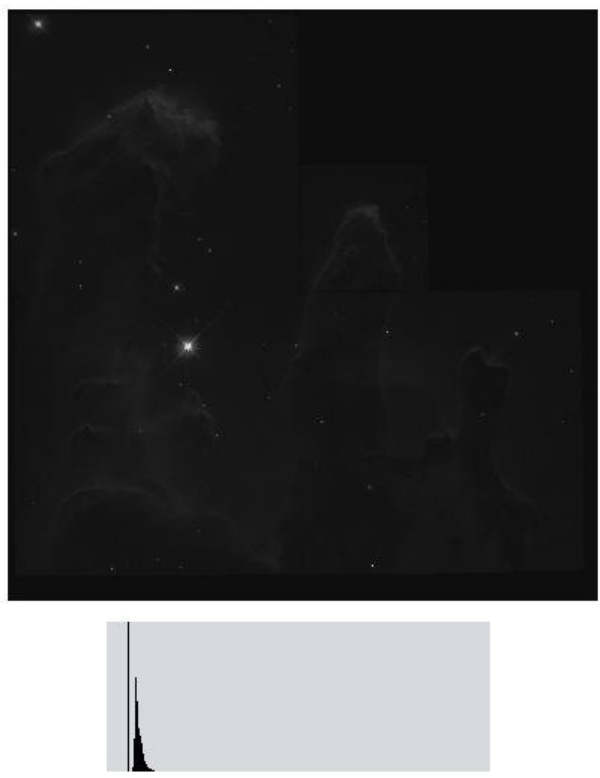

(b)
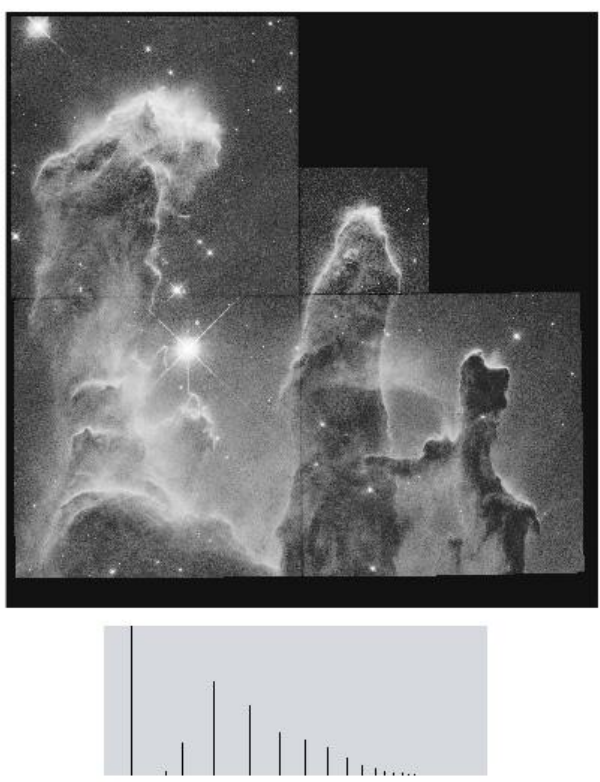

Figura 8. (a) Imagem original (b) Imagem equalizada e seus respectivos histogramas.

Com as três imagens representando três diferentes filtros de comprimento de onda, é possível obter uma imagem colorida contendo as características das três imagens em níveis de cinza.

É atribuído para cada canal do RGB (vermelho, verde e azul) sua respectiva intensidade de cinza a partir do filtro escolhido para aquela cor, dando origem a uma nova imagem colorida.

No exemplo mostrado na Figura 9, a imagem (a) foi capturada com um filtro de $673 \mathrm{~nm}$ e está representando a cor vermelha, a imagem (b), com um filtro de $656 \mathrm{~nm}$ está representando a cor verde e a imagem (c), com um filtro de $502 \mathrm{~nm}$, está representando a cor azul.

\subsection{Obtenção e representação do espectro astronômico}

Para obter o conjunto de dados com as intensidades do espectro foi utilizado o mesmo procedimento adotado na obtenção dos dados da imagem. A estrutura do espectro contém valores reais que representam a intensidade de brilho referente a uma faixa de comprimento de onda, como pode ser observado no gráfico de espectro mostrado na Figura 10.

Os valores referentes aos comprimentos de onda no gráfico são obtidos com a Equação 1:

$$
\lambda=10^{(\text {COEFF } 0+C O E F F 1 * i)}
$$


sendo que $i$ denota a quantidade de pontos no eixo $x$ começando de 0 , o valor do COEFFO e COEFF1 estão no cabeçalho do arquivo FITS.

Com o conhecimento desses valores, utilizou-se a biblioteca "JFreeChart" para plotar o gráfico do espectro.

(a)

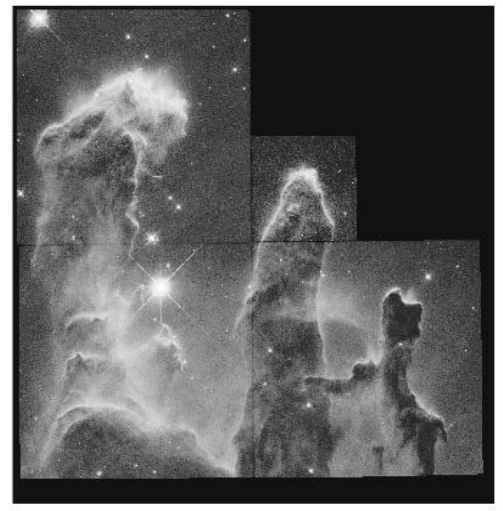

(c)

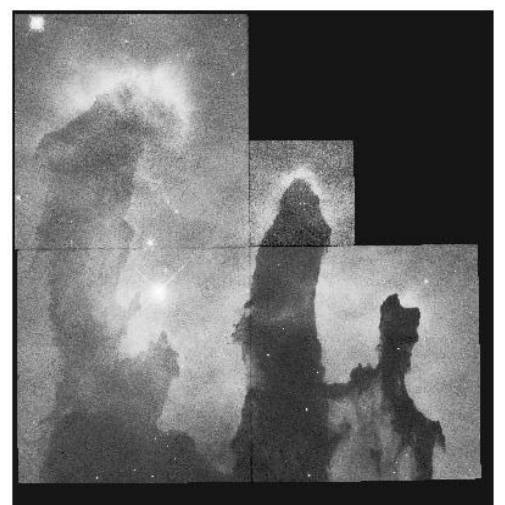

(b)

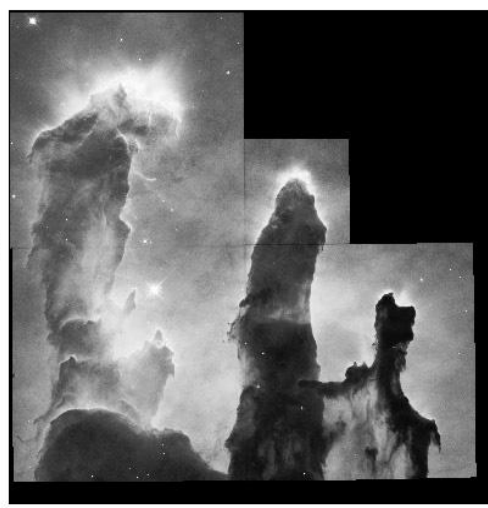

(d)

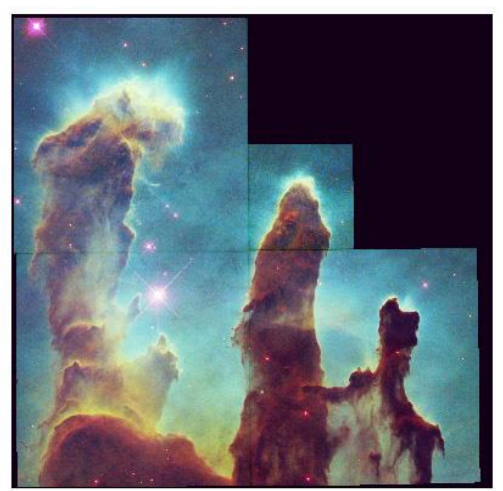

Figura 9. Imagem colorida (d) obtida através da união dos filtros (a),(b) e (c).

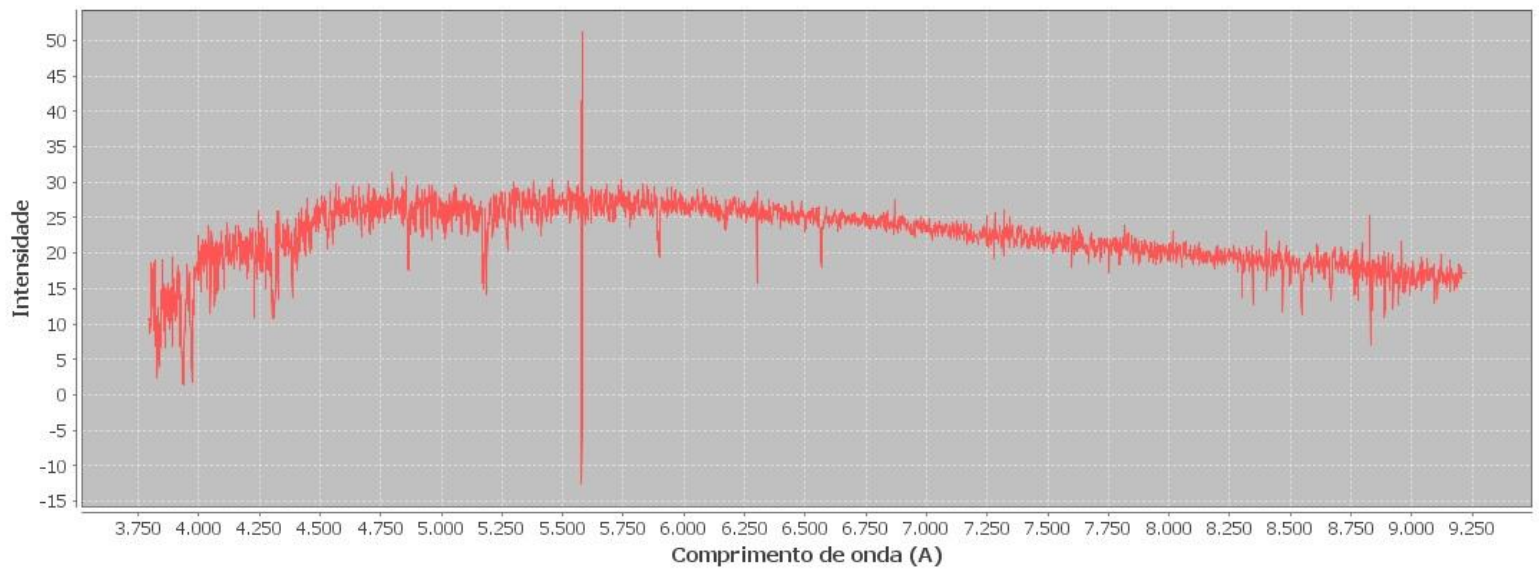

Figura 10. Gráfico de um espectro astronômico. 


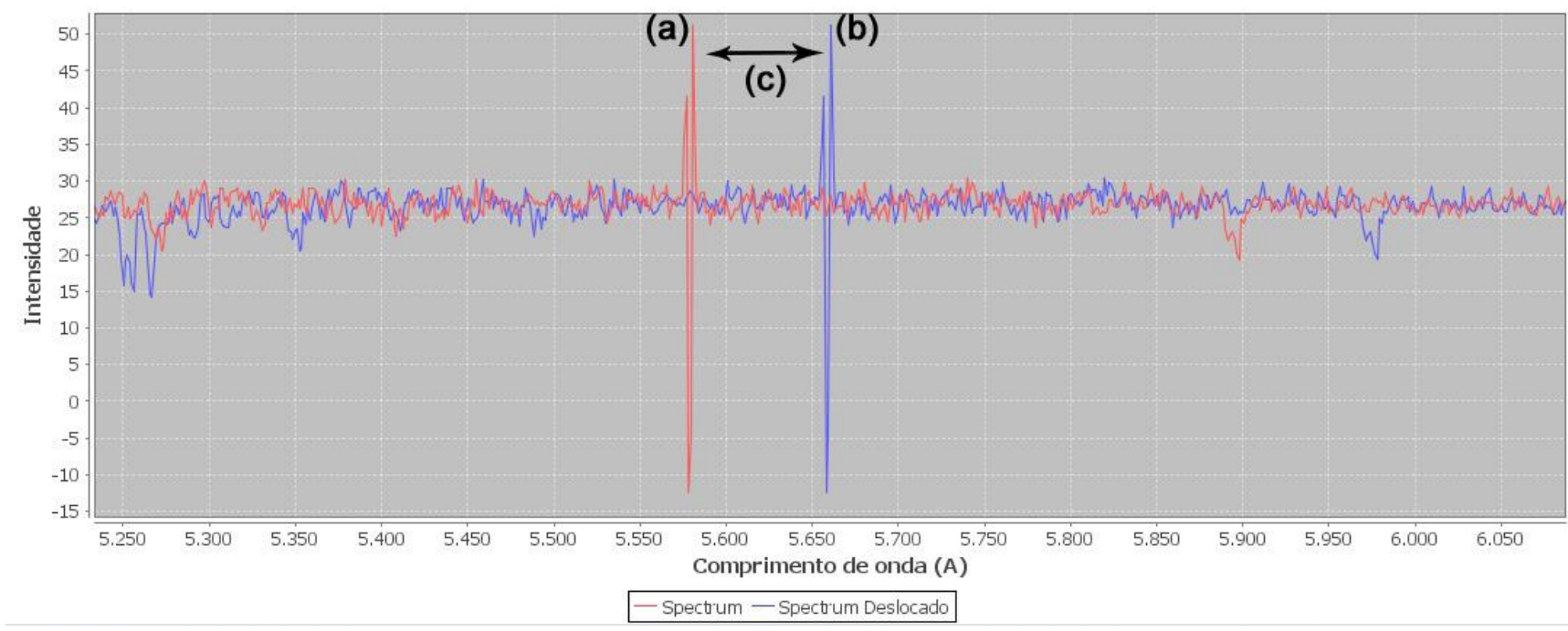

Figura 11. Espectro em repouso (vermelho) e o mesmo espectro deslocado (azul).

Para calcular a velocidade com que o objeto está se movimentando, foi utilizada a Equação 2.

velocidade $=c^{*}$ velocidade_da_luz / $a$

sendo $a$ o valor do comprimento de onda em Angstrom (1 Angstrom $=0,1 \mathrm{~nm}$ ) do espectro em repouso, $c$ é a diferença entra $a$ e $b$ que é igual a quantidade deslocada, velocidade da luz é $300.000 \mathrm{~km} / \mathrm{s}$.
Para identificar elementos presentes no objeto, foi traçada uma linha correspondente ao comprimento de onda de emissão do elemento para possibilitar uma melhor análise visual (Figura 12).

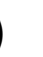




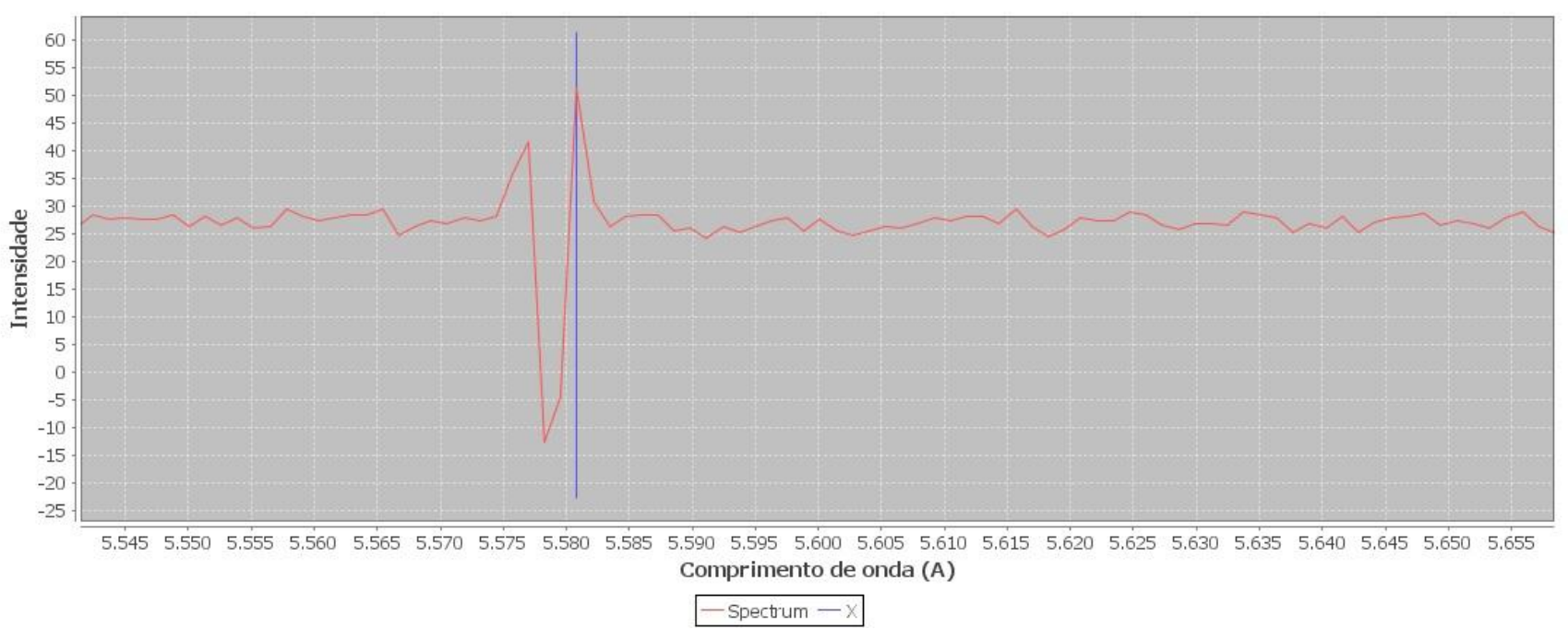

Figura 12. Linha azul representando o pico de emissão referente ao elemento buscado.

\section{EXPERIMENTOS E RESULTADOS}

Na Figura 13 é mostrado o resultado da imagem colorida utilizando as imagens originais, sem nenhum processamento para melhorar os detalhes.

Note que nessas imagens não há nenhum processamento, são mostradas exatamente como foram capturadas. O objeto mostrado é uma nebulosa que está a milhões de anos-luz da Terra, onde não há muita presença de luz, por isso elas são escuras.

As imagens (a), (b) e (c) foram obtidas em Nasa (2013). 
(a)

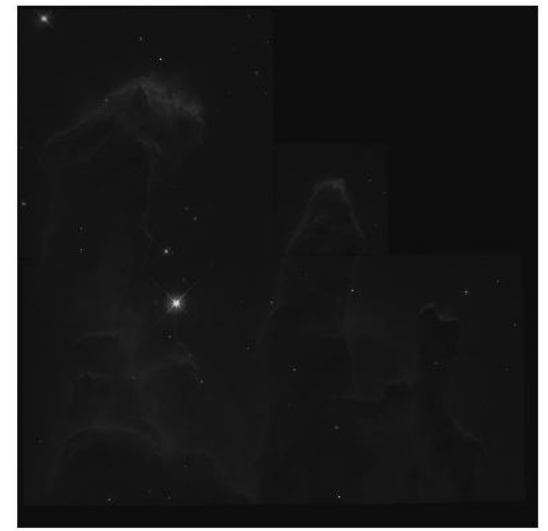

(c)

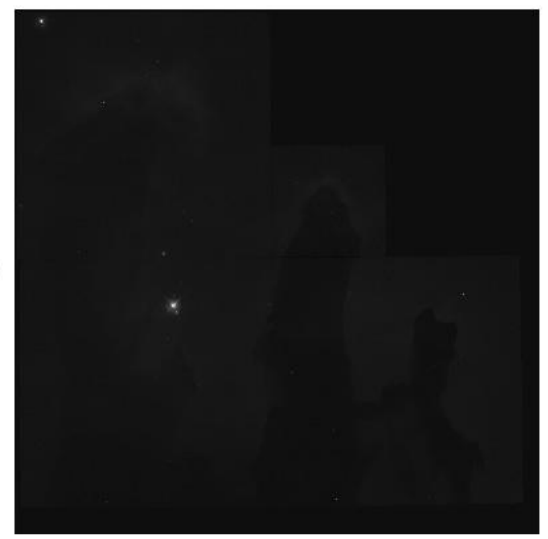

(b)

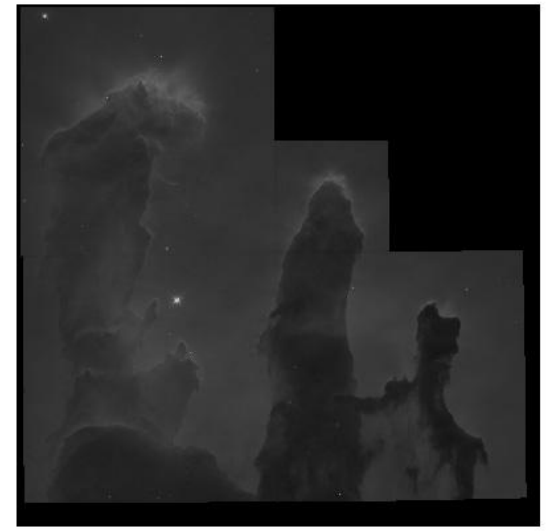

(d)

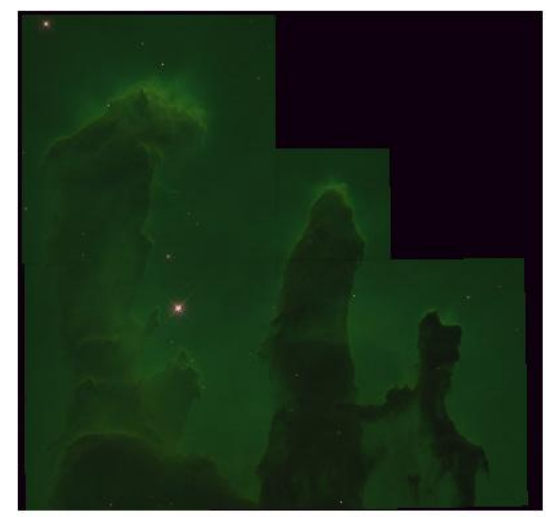

Figura 13. Imagem colorida (d) obtida através da união dos filtros (a), (b) e (c).

Com a utilização de três filtros, todos capturados com filtros de raios- $X$ (comprimento de onda médio de $3 \mathrm{~nm}$ ), foi possível criar uma imagem que seria invisível aos olhos, conforme mostrado na Figura 14, resultado do processamento efetuado com as imagens (a), (b), (c), retiradas em CHANDRA, (2013). 
(a)

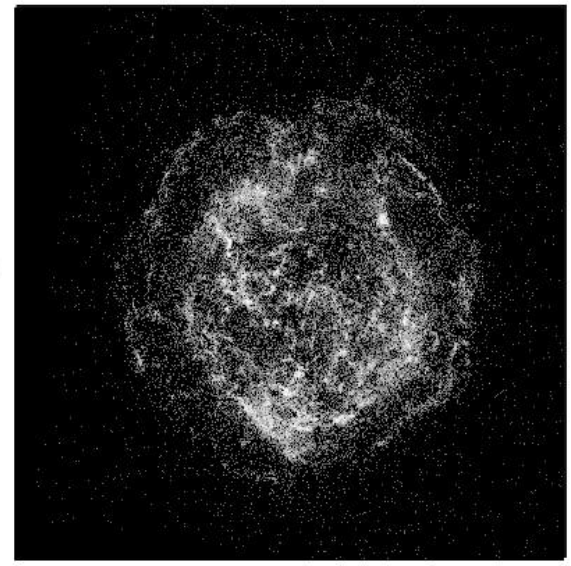

(c)

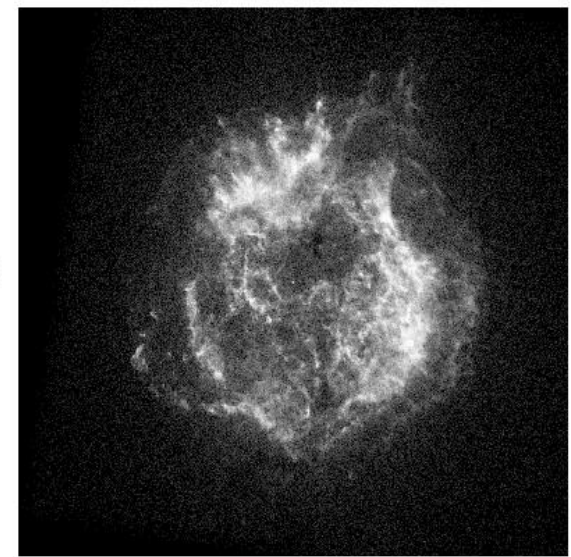

(b)

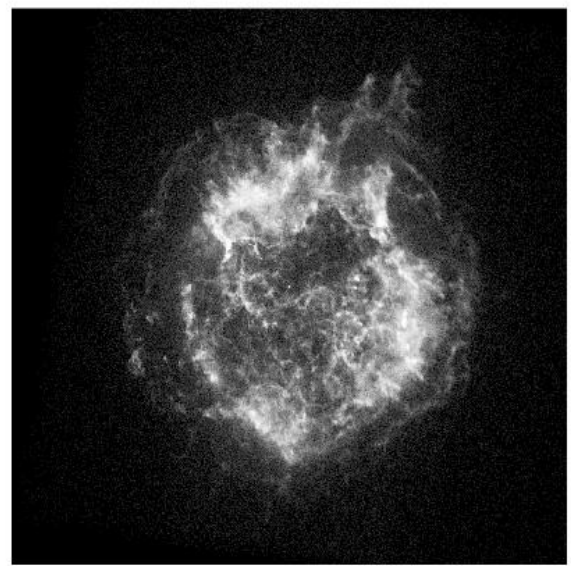

(d)

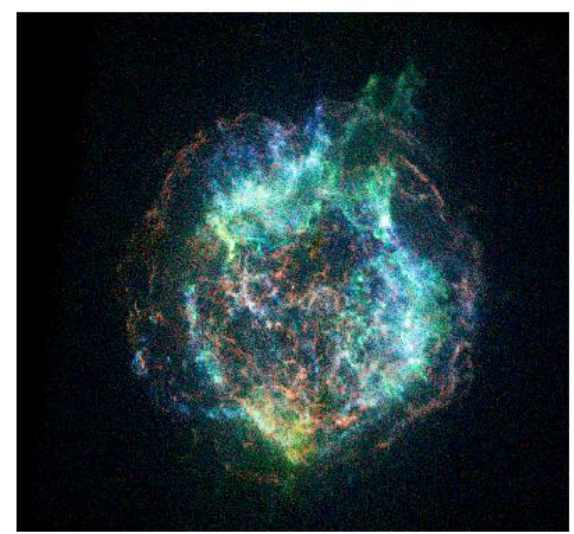

Figura 14. Imagem colorida (d) obtida através de três filtros de raios-X.

Se houvesse um deslocamento de onda de 60 Angstroms para a esquerda, significaria que o objeto está se aproximando do observador a uma velocidade de 4727,945 $\mathrm{km} / \mathrm{s}$, como mostrado na Figura 15. Pode-se comparar com o exemplo de um trem se aproximando do observador, quando o mesmo está se aproximando, o som é agudo, as ondas sonoras chegam até o observador com um menor comprimento do que se o trem estivesse em repouso. Quando o trem está se distanciando, o som é mais grave, pois as ondas chegam até o observador com um maior comprimento de onda, essa é a definição do efeito Doppler. 


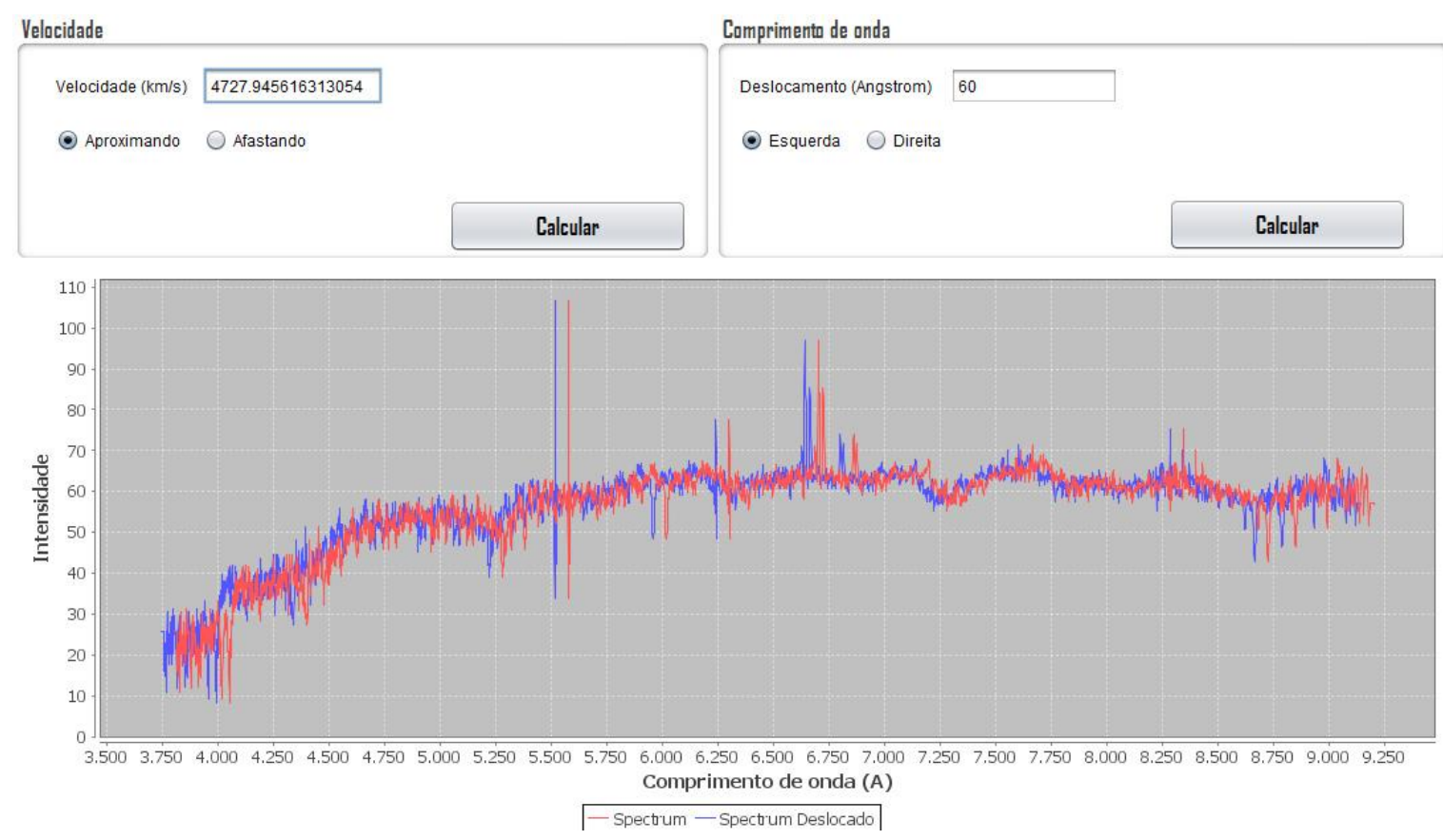

Figura 15. Espectro deslocado para simular o movimento do objeto.

\section{CONCLUSÕES}

Constatou-se que a ferramenta gerada neste trabalho realizou a função esperada, ou seja, mostrou como são obtidas as imagens astronômicas coloridas com falsa cor e o motivo de não serem originalmente colorida. Com a imagem é possível obter apenas informações referentes a um único comprimento de onda (como a presença de hidrogênio em uma nebulosa, por exemplo) para cada imagem (o filtro permite a passagem de um determinado comprimento de onda), ou seja, a imagem carrega consigo informações limitadas. Uma vantagem de usar filtros e obter imagens em níveis de cinza, é a captura de imagens a partir de comprimentos de onda invisíveis aos olhos humanos, facilitando o estudo por meio da imagem.
O espectro eletromagnético abrange um grande intervalo contendo muitos comprimentos de onda em um mesmo gráfico, possibilitando, assim, várias análises, como o movimento, a composição e a temperatura.

Uma alternativa para um trabalho futuro é realizar análises mais avançadas por meio dos espectros.

\section{REFERÊNCIAS}

BROSIUS, Jeffrey W. How astronomers use spectra to learn about the sun and other stars. [S.I.]: National Aeronautics and Space Administration, Goddard Space Flight Center, 1997.

CHANDRA. X-Ray Observatory. NASA's flagship mission for X-ray astronomy. Disponível em: < http://chandra. harvard.edu/ >. Acesso em: 11 set. 2013.

HUBBLE. Meaning of Color. Disponível em:<http://hubblesite.org/gallery/behind_th 
e_pictures/meaning_of_color/rgb.php $>$. Acesso em: 11 abr. 2013.

ITOKAZU, A. G. 1609: da astronomia tradicional ao nascimento da astrofísica. Cienc. Cult., São Paulo, v. 61 n. 4, 2009. Disponível em: <http://cienciaecultura. bvs.br/scielo.php?script=sci_arttext\&pid=S00 09-67252009000400014\&lng=pt\&nrm=iso $>$. Acesso em: 12 abr. 2013.

NASA. Definition of the flexible image transport system (FITS), v. 3, jul., 2008. Disponível em: <http://heasarc.gsfc.nasa. gov/docs/heasarc/fits/java/v1.0/FitsLib.pdf> . Acesso em: 26 fev. 2013.

NOGUEIRA, E. D. A.; VAZ, M. S. M.; SOUZA, L. Um padrão de metadados para descrição de imagens astronômicas do tipo FITS. Recen, Guarapuava, v. 12, n. 1, jan/jun., 2010.
OLIVEIRA FILHO, K. S.; SARAIVA, M. F. O. Astronomia e astrofísica. 2. ed. São Paulo: Ed. Liv. da Física, 2004.

STEINER, J. E. A origem do universo. Estudos Avançados, São Paulo, v. 20, n. 58, dec. 2006. Disponivel em: http://www.scielo.br/ scielo.php?script=sci_arttext $\&$ pid $=$ S010340142006000300022\&lng=en\&nrm=iso. Acesso em: 15 dec. 2013.

TENNYSON, Jonathan. Astronomical spectroscopy: an introduction to the atomic and molecular physics of astronomical spectra. Imperial College Press Advanced Physics Texts, v. 2, 2005.

WELLS, D. C.; GREISEN, E. W.; HAETEM, R. H. FITS: a flexible image transport system. Astron. Astrophys. Sup., p. 363-370, 1981. 